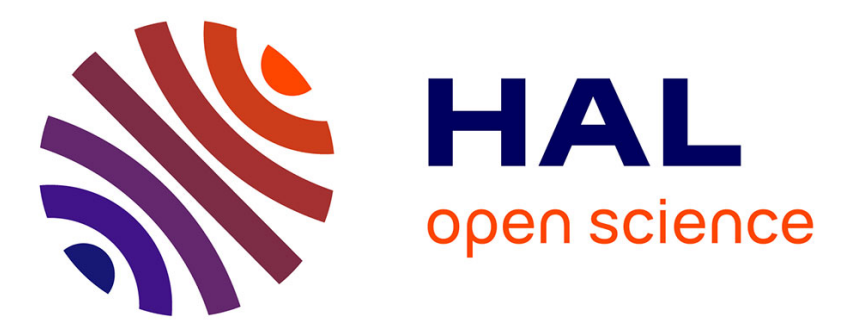

\title{
Clean surface studies by photoelectron diffraction analysed within a single scattering theory
}

D. Sébilleau, G. Tréglia, M.C. Desjonquères, D. Spanjaard, C. Guillot, D. Chauveau, J. Lecante

\section{- To cite this version:}

D. Sébilleau, G. Tréglia, M.C. Desjonquères, D. Spanjaard, C. Guillot, et al.. Clean surface studies by photoelectron diffraction analysed within a single scattering theory. Journal de Physique, 1988, 49 (2), pp.227-236. 10.1051/jphys:01988004902022700 . jpa-00210688

\section{HAL Id: jpa-00210688 https://hal.science/jpa-00210688}

Submitted on 1 Jan 1988

HAL is a multi-disciplinary open access archive for the deposit and dissemination of scientific research documents, whether they are published or not. The documents may come from teaching and research institutions in France or abroad, or from public or private research centers.
L'archive ouverte pluridisciplinaire HAL, est destinée au dépôt et à la diffusion de documents scientifiques de niveau recherche, publiés ou non, émanant des établissements d'enseignement et de recherche français ou étrangers, des laboratoires publics ou privés. 
Classification

Physics Abstracts

$61.14 \mathrm{D}-61.14 \mathrm{~F}-68.20-79.60 \mathrm{C}$

\title{
Clean surface studies by photoelectron diffraction analysed within a single scattering theory
}

\author{
D. Sébilleau $\left({ }^{1,2}\right)$, G. Tréglia $\left({ }^{3}\right)$, M. C. Desjonquères $\left({ }^{1}\right)$, D. Spanjaard $\left({ }^{3}\right)$, C. Guillot \\ $\left({ }^{1,2}\right)$, D. Chauveau $\left({ }^{1,2}\right)$ and J. Lecante $\left({ }^{1,2}\right)$ \\ ( $\left.{ }^{1}\right)$ IRF/D.Ph.G/PAS Centre d'Etudes Nucléaires de Saclay, 91191 Gif-sur-Yvette Cedex, France \\ $\left({ }^{2}\right)$ Laboratoire pour l'Utilisation du Rayonnement Electromagnétique (CNRS-CEA-MEN), Bât. 209D, \\ 91405 Orsay Cedex, France \\ $\left({ }^{3}\right)$ Laboratoire de Physique des Solides, Bât. 510, Université Paris-Sud, 91405 Orsay Cedex, France
}

(Reçu le 17 juillet 1987, accepté le 13 octobre 1987)

\begin{abstract}
Résumé. - Nous montrons qu'il est suffisant de traiter la diffraction de photoélectrons dans un formalisme de diffusion simple pour rendre compte de la dépendance azimutale observée de l'intensité photoémise par les niveaux $4 \mathrm{f}_{712}$ de $\mathrm{W}(100)$, et ce, même dans la gamme des basses énergies cinétiques pour lesquelles les émissions de volume et de surface sont bien séparées. Nous démontrons aussi qu'il est hasardeux de remplacer les fonctions d'onde $f$ exactes par des fonctions $s$, même si cela peut conduire à de bons résultats dans quelques cas particuliers. Enfin, la comparaison entre résultats expérimentaux et théoriques concernant la reconstruction de W(100) permet d'envisager l'utilisation de la diffraction de photoélectrons comme outil d'étude de possibles réarrangements des atomes des surfaces propres.
\end{abstract}

Abstract. - We show that a single scattering treatment of photoelectron diffraction is sufficient to account for the observed azimuthal dependence of the intensity of the W(100) $4 \mathrm{f}_{7 / 2}$ photoemission, even in the low kinetic energy range for which bulk and surface emissions are well separated. We also demonstrate that replacing the exact $f$ wave functions by an $s$ one is quite hazardous even though it might give good results in some cases. Finally, in view of the agreement between experimental and theoretical results on the reconstruction of W(100), we can be optimistic in the use of photoelectron diffraction to study clean surface atom rearrangements.

\section{Introduction.}

Core level photoelectron spectroscopy is a very sensitive probe of the chemical environment of the atom which undergoes the optical excitation. Among the data available from this type of experiment, two quantities have been more particularly investigated: the variation of the intensity of the lines with the angle of observation and the photon energy (photoelectron diffraction: $\mathrm{PhD}$ ) [1-3] and the binding energy shift of the surface levels relative to the bulk one [4-13]. Historically, Liebsch was the first to prove that photoelectron diffraction could be used for the determination of the surface structure of adsorbates [14]. Indeed, the symmetry of the adsorption site is directly given by the symmetry of the azimuthal pattern [1]. The precise determination of distances requires a comparison of the experimental

data with the calculated patterns. Whereas the first calculations have been performed using a multiple scattering theory [14], Kono et al. [15] have shown that a single scattering model was sufficient to explain the experimental data, at least for kinetic energies larger than $200 \mathrm{eV}$. The study of clean surfaces, which requires kinetic energies in the range 20-200 eV, is much more complicated since there are several types of emitters : surface, first underlayer, ..., bulk atoms. However, these emitters having their own geometrical environment, their core level lines should not appear strictly at the same energy. Therefore, if the various lines can be resolved, one should be able to obtain experimental patterns specific of each type of emitter. The core level binding energy shift between surface and bulk atoms 
being of less than $1 \mathrm{eV}$, such surface studies will only be possible for very narrow lines such as the $4 \mathrm{f} \mathrm{levels}$ of transition metals of the $5 \mathrm{~d}$ series [4-13]. One can wonder yet whether the single scattering treatment of the diffraction is still adequate for such small kinetic energies. The aim of the present paper is first to check the validity of these calculations in the particular case of azimuthal patterns of the $4 \mathrm{f}$ levels of $W(100)$ and then to investigate its sensitivity to atomic displacements (relaxation, reconstruction). In view of the good agreeement between experimental and theoretical results, we also give the calculated curves for the other two low index faces of this metal.

\section{Single scattering formalism.}

If indeed Kono et al. $[3,15]$ were the first to use a single scattering treatment to interpret their $\mathrm{PhD}$ experiments, this model had already been used by Mc Donnell et al. [16] to study the angular anisotropy of the Auger emission. The corresponding theoretical model was first developed by Liebsch [14] and
Lee [17] in the particular case of a spherical $s$ initial state and then exterided to the case of any initial state by Fujikawa [18]. Here, we will first derive the formula of Fujikawa in a slightly different manner. Then, although we are interested in $\mathrm{f}$ initial states, we will show how it simplifies in the case of an s state to recover the Kono et al. formula. Actually, we will also use this last formula to check the validity of the Kono et al's conjecture according to which it can account for the emission from core levels belonging to filled shells which have a spherically symmetric charge distribution [15].

Let us now briefly give the main steps of the derivation of the general formula. The photoelectron wave function $\psi(\mathbf{R})$ at the detector located at $\mathbf{R}$ is given by :

$$
\psi(\mathbf{R}) \propto \int \mathrm{d}_{3} \mathbf{r} G(\mathbf{R}, \mathbf{r}) \mathbf{p} \cdot \mathbf{A} \psi_{i}(\mathbf{r})
$$

where $\psi_{i}(\mathbf{r})$ is the core orbital of the photoexcited electron, $\mathbf{A}$ the vector potential, $\mathbf{p}$ the momentum operator and $G(\mathbf{R}, \mathbf{r})$ is the propagator of the emitted electron :

$$
G(\mathbf{R}, \mathbf{r})=G_{0}(\mathbf{R}, \mathbf{r})+\sum_{j} \iint \mathrm{d}_{3} \mathbf{r}^{\prime} \mathrm{d}_{3} \mathbf{r}^{\prime \prime} g_{0}\left(\mathbf{R}, \mathbf{r}^{\prime}\right) T_{j}\left(\mathbf{r}^{\prime}, \mathbf{r}^{\prime \prime}\right) G_{0}\left(\mathbf{r}^{\prime \prime}, \mathbf{r}\right)
$$

where $G_{0}$ is the propagator of the photoexcited electron for an isolated emitter, $T_{j}$ is the $T$-matrix describing the diffusion of the electron by the neighbour $j$ at distance $R_{j}$ from the emitter and $g_{0}$ is the free electron propagator ; they are given, in Hartree units, for $R>r^{\prime}$ and $r^{\prime \prime}>r$ and outside the range of the potential of the emitter, by :

$$
\begin{aligned}
& G_{0}\left(\mathbf{r}^{\prime \prime}, \mathbf{r}\right)=-2 i k \sum_{\ell_{m}} Y_{\ell_{m}}\left(\hat{r}^{\prime \prime}\right) Y_{\ell_{m}}^{*}(\hat{r}) h_{\ell}^{(1)}\left(k r^{\prime \prime}\right) \mathrm{e}^{i \delta \ell_{\ell}} \mathcal{R}_{\ell}(k r) \\
& g_{0}\left(\mathbf{R}, \mathbf{r}^{\prime}\right)=-2 k \sum_{\ell_{m}} Y_{\ell_{m}}(\hat{R}) Y_{\ell_{m}}^{*}\left(\hat{r}^{\prime}\right) \frac{\mathrm{e}^{i\left(k R-\ell \frac{\pi}{2}\right)}}{k R} j_{\ell}\left(k r^{\prime}\right)
\end{aligned}
$$

where $Y_{\ell_{m}}$ are the spherical harmonics, $j_{\ell}$ the spherical Bessel functions, $h_{\ell}^{(1)}$ the Hankel functions of the first kind, $R_{\ell}(k r)$ the regular solution of the radial Schrödinger equation of the emitter atom, $\delta_{\ell}$ the corresponding phase-shift and $k$ the wave vector. With these notations, $G_{0}(\mathbf{R}, \mathbf{r})$ for $R \gg r$ is obtained by using the first term of the asymptotic development of $h_{\ell}^{(1)}(k R) \simeq \frac{-i \mathrm{e}^{i\left(k R-\ell \frac{\pi}{2}\right)}}{k R}$. To treat the diffusion by a spherical potential centred at site $j$, it is convenient to use functions centred at this site. First we take advantage of the invariance of $g_{0}$ with respect to the translation $\mathbf{R}_{j}$, i.e. $g_{0}\left(\mathbf{R}, \mathbf{r}^{\prime}\right)=g_{0}\left(\mathbf{R}-\mathbf{R}_{j}, \mathbf{r}^{\prime}-\mathbf{R}_{j}\right)$ which can be written, since $R \gg R_{j}$ :

$$
g_{0}\left(\mathbf{R}, \mathbf{r}^{\prime}\right) \simeq-2 \sum_{\ell_{m}}(-i)^{\ell} Y_{\ell_{m}}(\hat{R}) \frac{\mathrm{e}^{+i k\left(R-\hat{R} \cdot \mathbf{R}_{j}\right)}}{R} Y_{\ell_{m}}^{*}\left(\widehat{r^{\prime}-R_{j}}\right) j_{\ell}\left(k\left|\mathbf{r}^{\prime}-\mathbf{R}_{j}\right|\right) .
$$

Then we rewrite the spherical wave $Y_{\ell_{m}}\left(\hat{r}^{\prime \prime}\right) h_{\ell}^{(1)}\left(k r^{\prime \prime}\right)$ outgoing from the emitter in terms of spherical waves incoming on the neighbour $j[19]$ :

$$
Y_{\ell m}\left(\hat{r}^{\prime \prime}\right) h_{\ell}^{(1)}\left(k r^{\prime \prime}\right)=\sum_{\ell^{\prime} m^{\prime}} \mathcal{G}_{\ell_{m \ell^{\prime} m^{\prime}}}^{j_{\ell^{\prime}}}\left(k\left|\mathbf{r}^{\prime \prime}-\mathbf{R}_{j}\right|\right) Y_{\ell^{\prime} m^{\prime}}\left(\widehat{r^{\prime \prime}-R_{j}}\right)
$$


with :

$$
\mathcal{S} \dot{k}_{m \ell^{\prime} m^{\prime}}=4 \pi \sum_{\ell^{\prime \prime} m^{\prime \prime}} i^{\ell-\ell^{\prime \prime}-\ell^{\prime}} h_{\ell^{\prime \prime}}^{(1)}\left(k R_{j}\right) Y_{\ell^{\prime \prime} m^{\prime \prime}}^{*}\left(\hat{R}_{j}\right) G\left(\ell m, \ell^{\prime \prime} m^{\prime \prime} \mid \ell^{\prime} m^{\prime}\right)
$$

where $G\left(\ell m, \ell^{\prime \prime} m^{\prime \prime} \mid \ell^{\prime} m^{\prime}\right)$ is the Gaunt coefficient given by :

$$
G\left(\ell m, \ell^{\prime \prime} m^{\prime \prime} \mid \ell^{\prime} m^{\prime}\right)=\int \mathrm{d} \Omega Y_{\ell m}(\hat{r}) Y_{\ell^{\prime \prime} m^{\prime \prime}}(\hat{r}) Y_{\hat{\ell}^{\prime} m^{\prime}}^{*}(\hat{r})
$$

Since the diffusion potential has a spherical symmetry, the $T$ operator only acts on the radial part of the incoming spherical wave. Then, taking into account the well-known relation :

$$
\iint r_{j}^{\prime 2} r_{j}^{\prime \prime 2} j_{\ell}\left(k r_{j}^{\prime}\right) T\left(r_{j}^{\prime}, r_{j}^{\prime \prime}\right) j_{\ell}\left(k r_{j}^{\prime \prime}\right) \mathrm{d} r_{j}^{\prime} \mathrm{d} r_{j}^{\prime \prime}=-\frac{\mathrm{e}^{i \delta_{\ell}^{j}} \sin \delta \dot{k}}{2 k}
$$

where $r_{j}^{\left({ }^{\prime}\right)}=\left|\mathbf{r}^{\prime\left({ }^{\prime}\right)}-\mathbf{R}_{j}\right|$, and $\delta \dot{b}$ is the phase shift due to scatterer $j$, one finds :

$$
\psi(\mathbf{R}) \propto \frac{-2 \mathrm{e}^{i k R}}{R} \sum_{\ell_{m}}\left\{(-i)^{\ell} Y_{\ell_{m}}(\hat{R})+i \sum_{j} \mathrm{e}^{-i k R \cdot \mathbf{R}_{j}} \sum_{\ell^{\prime} m^{\prime}}(-i)^{\ell^{\prime}} Y_{\ell^{\prime} m^{\prime}}(\hat{R}) \mathcal{S}_{\xi_{\ell^{\prime} m^{\prime}}} \mathrm{e}^{i \delta_{\ell^{\prime}}^{j}} \sin \delta \dot{k}^{\prime}\right\} M_{\ell_{m}, i}
$$

with

$$
M_{\ell m, i}=\int \mathrm{d}_{3} \mathbf{r} Y_{\ell m}^{*}(\hat{r}) \mathcal{R}_{\ell}(k r) \mathbf{p} \cdot \mathbf{A} \psi_{i}(\mathbf{r}) \mathrm{e}^{i \delta_{\ell}}
$$

In the dipole approximation and for an initial state $\psi_{i}(\mathbf{r})=R_{\ell_{i}}(r) Y_{\ell_{i} m_{i}}(\hat{r})$

$$
M_{\ell_{m, i}}=M_{\ell_{m}, \ell_{i} m_{i}} \propto \int Y_{l m}^{*}(\hat{r}) \mathcal{R}_{\ell}(k r) \hat{\varepsilon} \cdot \hat{r} R_{\ell_{i}}(r) Y_{\ell_{i} m_{i}}(\hat{r}) r^{3} \mathrm{~d} r \mathrm{~d} \Omega
$$

Then, making use of the relationship

with

$$
\begin{aligned}
& \hat{\varepsilon} \cdot \hat{r}=\frac{4 \pi}{3} \sum_{m=-1}^{m=+1} Y_{1 m}^{*}(\hat{\varepsilon}) Y_{1 m}(\hat{r}) \\
& M_{\ell_{m}, \ell_{i} m_{i}} \propto(-i)^{\ell} \mathrm{e}^{i \delta_{\ell} \boldsymbol{l}^{m^{\prime}=+1}} \sum_{m^{\prime}=-1}^{Y_{1 m^{\prime}}}(\hat{\varepsilon}) G\left(l_{i} m_{i}, 1 m^{\prime} \mid \ell m\right) \tilde{\rho}_{k}\left(\ell, \ell_{i}\right)
\end{aligned}
$$

and finally :

$$
\psi(\mathbf{R}) \propto \frac{\mathrm{e}^{i k R}}{R} \sum_{\ell_{m}} M_{\ell_{m}, \ell_{i} m_{i}}\left\{Y_{\ell_{m}}(\hat{R})+i \sum_{j} \mathrm{e}^{-i k R \cdot \mathbf{R}_{j}} \sum_{\ell^{\prime} m^{\prime}}(-i)^{\ell^{\prime}-\ell} Y_{\ell^{\prime} m^{\prime}}(\hat{R}) \mathfrak{S}_{m_{m} \ell^{\prime} m^{\prime}} \mathrm{e}^{i \delta_{\ell^{\prime}}^{j}} \sin \delta \dot{\phi^{\prime}}\right\}
$$

Note that the Gaunt coefficient in $M_{\ell_{m}, \ell_{i} m_{i}}$ is 0 except for $\ell=\ell_{i}-1$ and $\ell=\ell_{i}+1$ which are then the only transitions allowed.

The photocurrent is given by :

$$
\frac{\mathrm{d} I}{\mathrm{~d} \Omega}=R^{2} \operatorname{Im}\left(\psi^{*}(\mathbf{R})\left|\frac{\partial \psi}{\partial r}\right|_{\mathbf{r}=\mathbf{R}}\right)
$$

which, using (Eq. (5)) leads to :

$$
\begin{aligned}
\frac{\mathrm{d} I}{\mathrm{~d} \Omega} \propto k & \mid \sum_{\ell_{m}} M_{\ell_{m}, \ell_{i} m_{i}}\left\{Y_{\ell_{m}}(\hat{R})+4 i \pi \sum_{j \neq 0} \mathrm{e}^{-i k R_{j} \cos \vartheta_{j}}\right. \\
& \left.\sum_{\ell^{\prime \prime} m^{\prime \prime}} Y_{\ell^{\prime \prime} m^{\prime \prime}}^{*}\left(\hat{R}_{j}\right) h_{\ell^{\prime \prime}}^{(1)}\left(k R_{j}\right)(-i)^{\ell^{\prime \prime}} \sum_{\ell^{\prime} m^{\prime}}(-1)^{\ell-\ell^{\prime}} Y_{\ell^{\prime} m^{\prime}}(\hat{R}) \mathrm{e}^{i \delta_{\ell^{\prime}}^{j}} \sin \delta \dot{k}, G\left(\ell m, \ell^{\prime \prime} m^{\prime \prime} \mid \ell^{\prime} m^{\prime}\right)\right\}\left.\right|^{2}
\end{aligned}
$$


where $\vartheta_{j}$ is the scattering angle, or angle between $\hat{R}$ and $\hat{R}_{j}$. Equation (13) is equivalent to the equations derived by Fujikawa [18] and Sagurton et al. [21]. This formula is the exact single scattering solution in a spherical wave description. It greatly simplifies if $k R_{j}$ is sufficiently large. Indeed, in this case, $h_{\ell^{\prime \prime}}^{(1)}\left(k R_{j}\right)$ can be replaced by the asymptotic development given above. Then, using the formula [22] :

$$
Y_{\ell_{m}}\left(\hat{R}_{j}\right) Y_{\ell^{\prime} m^{\prime}}^{*}\left(\hat{R}_{j}\right)=\sum_{\ell^{\prime \prime} m^{\prime \prime}} Y_{\ell^{\prime \prime} m^{\prime \prime}}^{*}\left(\hat{R}_{j}\right) G\left(\ell m, \ell^{\prime \prime} m^{\prime \prime} \mid \ell^{\prime} m^{\prime}\right)
$$

and the condition that $\left(\ell+\ell^{\prime \prime}-\ell^{\prime}\right)$ should be even, one finds :

$$
\frac{\mathrm{d} I}{\mathrm{~d} \Omega} \propto k\left|\sum_{\ell_{m}} M_{\ell_{m}, \ell_{i} m_{i}}\left\{Y_{\ell_{m}}(\hat{R})+\sum_{j \neq 0} \frac{\mathrm{e}^{i k R_{j}\left(1-\cos \vartheta_{j}\right)}}{R_{j}} f\left(\boldsymbol{\vartheta}_{j}\right) Y_{\ell_{m}}\left(\hat{R}_{j}\right)\right\}\right|^{2}
$$

in which $f\left(\vartheta_{j}\right)$ is the usual scattering factor :

$$
f\left(\vartheta_{j}\right)=\frac{4 \pi}{k} \sum_{\ell^{\prime} m^{\prime}} \mathrm{e}^{i \delta_{\ell^{\prime}}^{j}} \sin \delta \dot{k^{\prime}} Y_{\ell^{\prime} m^{\prime}}(\hat{R}) Y_{\ell^{\prime} m^{\prime}}^{*}\left(\hat{R}_{j}\right)
$$

which is equivalent to Fujikawa formula.

Note that this formula is valid for any initial state but can be still simplified if one considers an $\mathrm{s}$ initial state $\left(\ell_{i}=0\right)$. Actually, in that case, the only possible transition is towards $\ell=1$ and the matrix element becomes :

$$
M_{1 m, 00} \propto-i \mathrm{e}^{i \delta_{1}^{0}} Y_{1 m}^{*}(\hat{\varepsilon}) \tilde{\rho}_{k}(1,0) .
$$

Using once again equation (10) one obtains the formula valid for a s initial state

$$
\frac{\mathrm{d} I}{\mathrm{~d} \Omega} \propto k \tilde{\rho}_{k}^{2}(1,0)\left|\left\{\hat{\varepsilon} \cdot \hat{R}+\sum_{j \neq 0} \hat{\varepsilon} \cdot \hat{R}_{j} \frac{\mathrm{e}^{+i k R_{j}\left(1-\cos \vartheta_{j}\right)} f\left(\vartheta_{j}\right)}{R_{j}}\right\}\right|^{2}
$$

which is the formula initially derived by Kono et al. [15].

3. Application to the study of the $4 f$ core level emission from $\mathbf{W}$ surfaces.

Let us now apply this formalism in the particular case of the $4 \mathrm{f}$ core levels $\left(\ell_{i}=3\right)$ of $\mathrm{W}$. Then, due to the above mentioned selection rule, the allowed final state has angular momentum $\ell=2$ or $\ell=4$. One can show that the latter is negligible in the energy range where the maximum surface sensitivity is obtained. This can be qualitatively understood if one assumes that the final state is a plane wave. Actually, in this case, the ratio of the radial matrix elements, which is given by :

$$
\frac{\tilde{\rho}_{k}(4,3)}{\tilde{\rho}_{k}(2,3)}=\frac{\int_{0}^{\infty} r^{3} j_{4}(k r) R_{3}(r) \mathrm{d} r}{\int_{0}^{\infty} r^{3} j_{2}(k r) R_{3}(r) \mathrm{d} r},
$$

can be estimated to be of the order $10^{-1}$ (see Appendix). Note however that this ratio is energy dependent so that the transition towards $\ell=4$ state can become non-negligible, and even predominant, at larger energies. Here, we will thus neglect this $\ell=4$ optical transition so that equation (15) can be rewritten :

$$
\begin{aligned}
\frac{\mathrm{d} I}{\mathrm{~d} \Omega} \propto & \sum_{m_{i}=-3}^{m_{i}=+3} k \mid \sum_{m=-2}^{m=+2} M_{2 m, 3 m_{i}}\left\{Y_{2 m}(\hat{R})+\right. \\
& \left.+\sum_{j \neq 0} \frac{\mathrm{e}^{i k R_{j}\left(1-\cos \vartheta_{j}\right)}}{R_{j}} f\left(\vartheta_{j}\right) Y_{2 m}\left(\hat{R}_{j}\right)\right\}\left.\right|^{2} .
\end{aligned}
$$

In order to calculate azimuthal patterns, for a given energy, the radial matrix element factorizes so that (19) becomes

$$
\begin{aligned}
& \left.\frac{\mathrm{d} I}{\mathrm{~d} \Omega} \propto \sum_{m_{i}=-3}^{m_{i}=+3}\right|_{m=-2} ^{m=+2} Y_{1}^{*}{ }_{m-m_{i}}(\hat{\varepsilon}) G\left(3 m_{i}, 1\left(m-m_{i}\right) \mid 2 m\right) \\
& \times\left.\left\{Y_{2 m}(\hat{R})+\sum_{j \neq 0} \frac{\mathrm{e}^{i k R_{j}\left(1-\cos \vartheta_{j}\right)}}{R_{j}} f\left(\vartheta_{j}\right) Y_{2 m}\left(\hat{R}_{j}\right)\right\}\right|^{2}
\end{aligned}
$$


We have performed the calculations for the three low index faces of $\mathrm{W}$ taking into account [16] :

- the inelastic effects as an isotropic attenuation $\mathrm{e}^{-\gamma L\left(L_{j}\right)}$ of the electronic amplitude, $(1 / 2 \gamma:$ electron mean free path, $L\left(L_{j}\right)$ : distance from emitter to the surface (via the $j$-th scatterer))

- and the thermal vibrations via a Debye-Waller factor.

We have taken the following parameters (see Fig. 1) :

- polar angle : $\theta=30^{\circ}$

- angle between the polarization vector $\hat{\varepsilon}$ and the surface normal : $\alpha=22^{\circ} 5$

- and photon energy : $h \nu=65 \mathrm{eV}$.

These parameters correspond to the experimental conditions in the particular case of W(100) (see Sect. 5). The scattering factor is calculated from the potential of Christensen and Feuerbacher [23], which is assumed to be the same for bulk and surface atoms. The surface potential barrier between vacuum and bulk is taken into account by allowing a slight refraction effect changing the emergence angle $\vartheta$ of the photoelectron inside the solid to $\boldsymbol{\vartheta}^{\prime}$ in vacuum according to the relation:

$$
\sin ^{2} \vartheta=\left(1+V_{0} / E_{k}\right) \sin ^{2} \vartheta^{\prime},
$$

where $V_{0}$ is the value of the inner potential $\left(V_{0}=-14 \mathrm{eV}\right)$ and $E_{k}$ is the kinetic energy of the electron inside the metal. In our calculations we limit the summations over $j$ in equation (20) to terms where $L_{j} \leqq \gamma^{-1}$. At the kinetic energies of the photoelectrons in our experiments $(\sim 30 \mathrm{eV})$, $\gamma^{-1} \simeq 10 \AA$ so that the emission comes mainly from the first layers. In practice, only relatively small clusters are needed in the calculations ( $\sim 33$ atoms) : we have checked that increasing the allowed values of $L_{j}$ beyond $\gamma^{-1}$, although it slightly changes the relative intensities of the structures, does not modify their angular positions. In a first step, the three surfaces are assumed to be unrelaxed and unreconstructed. Since the bulk (B), subsurface

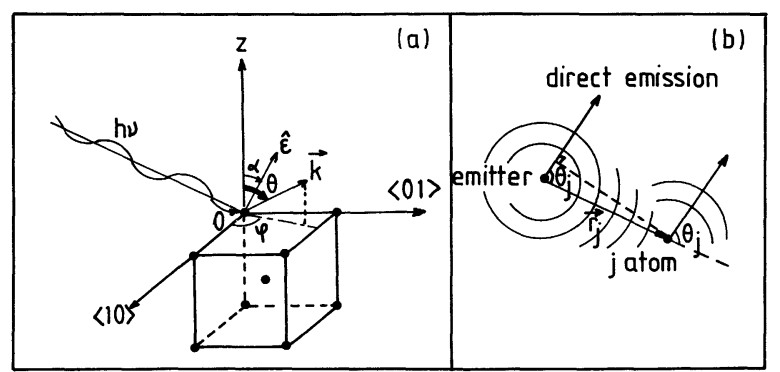

Fig. 1. - Schematic representations of the experimental geometry defining the angles used in the main text (a) and of the single scattering model (b).
$\left(S_{2}\right)$ (at least for (111) and (100) faces) and surface $\left(\mathrm{S}_{1}\right)$ emissions can be distinguished experimentally, we have plotted separately the azimuthal patterns for the $\left(B+S_{1}+S_{2}\right), \quad B$ and $S_{1}$ emissions in figures $2\left(a, a^{\prime}, a^{\prime \prime}\right), 2\left(b, b^{\prime}, b^{\prime \prime}\right)$ and $2\left(c, c^{\prime}, c^{\prime \prime}\right)$ respectively. $\varphi=0^{\circ}$ corresponds to the $\langle 001\rangle$, $\langle 001\rangle,\langle 1 \overline{1} 0\rangle$ directions for the (110), (100), (111) surfaces respectively. Obviously, these patterns reflect the symmetry of the corresponding faces. The surface emission is less anisotropic than the bulk one. Actually, under these experimental conditions, most of the atoms giving rise to forward scattering (which is predominant) are suppressed for surface emitters. Let us point out that, although equation (20) has been first derived a few years ago [18], it had never been applied in practice to an experimental case with $\ell_{i} \neq 0$, at least to our knowledge. Indeed, most experiments have been performed on s core levels, except for the $\mathrm{Cu} p$ levels. However, in this last case, Kono et al. [15], arguing that these p-levels being filled have a spherical charge distribution, have proposed to still use the formula valid for an $s$ initial state (Eq. 18). This approximation enabled these authors to interpret satisfactorily their experiments. More recently, Sébilleau et al. [24] have used the same approximation in the case of the $\mathrm{W}(100) 4 \mathrm{f}$ core levels with the same success. In view of equations (18) and (20) which look quite different, one can wonder to what extent this approximation is general. In order to

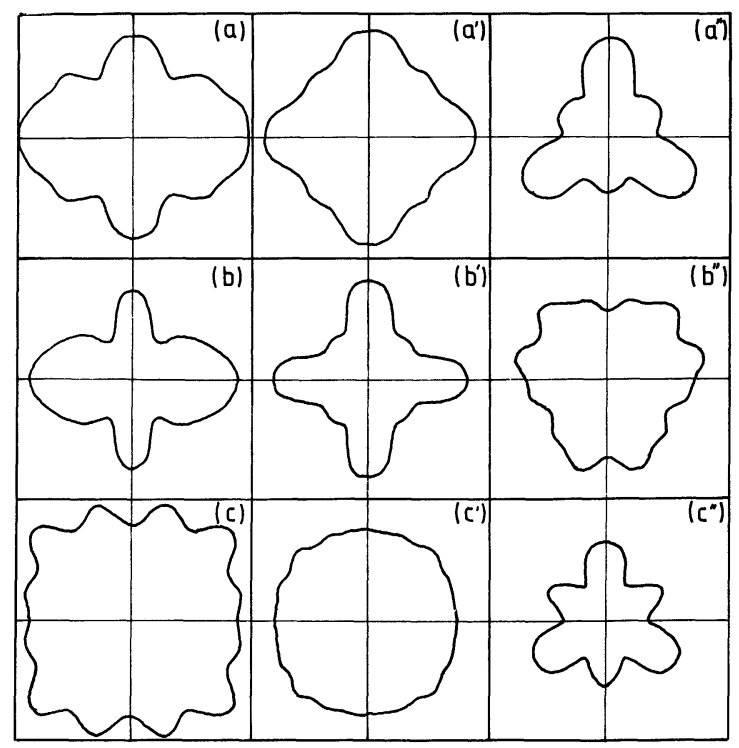

Fig. 2. - Polar plots of the theoretical azimuthal dependence of $\mathrm{W}(110):(a, b, c), W(100):\left(a^{\prime}, b^{\prime}, c^{\prime}\right)$ and $\mathrm{W}(111):\left(a^{\prime \prime}, b^{\prime \prime}, c^{\prime \prime}\right) 4 f_{712}$ core photoelectron intensities for the total $\left(a, a^{\prime}, a^{\prime \prime}\right)$, bulk $\left(b, b^{\prime}, b^{\prime \prime}\right)$ and surface $\left(c, c^{\prime}, c^{\prime \prime}\right)$ emissions, calculated from Fujikawa formula (neglecting the $\mathrm{f} \rightarrow \mathrm{g}$ transition) for the following conditions : $\boldsymbol{\theta}=30^{\circ}$, $\alpha=22^{\circ} 5, h \nu=65 \mathrm{eV}$. 
check this point, we have also calculated the azimuthal patterns for the two other low index faces of $W$ within this approximation. The corresponding results are shown in figure 3 . One sees at first glance that although the agreement between figure 2 and figure 3 is good in the case of $W(100)$, it is less satisfactory for W(110) and non existent for W(111). In view of these results, one can conclude that it is more advisable to use the exact formula (20), even for filled inner shells, although in some particular cases, the simplified formula (18) might give satisfactory results.

Finally, let us remark that the azimuthal patterns are very sensitive to the experimental conditions. To illustrate this point, we give in figure 4 the azimuthal patterns of $4 \mathrm{f}$ levels of W(110) for an other set of parameters [25] : $\theta=45^{\circ}, \alpha=20^{\circ}, h \nu=70 \mathrm{eV}$.

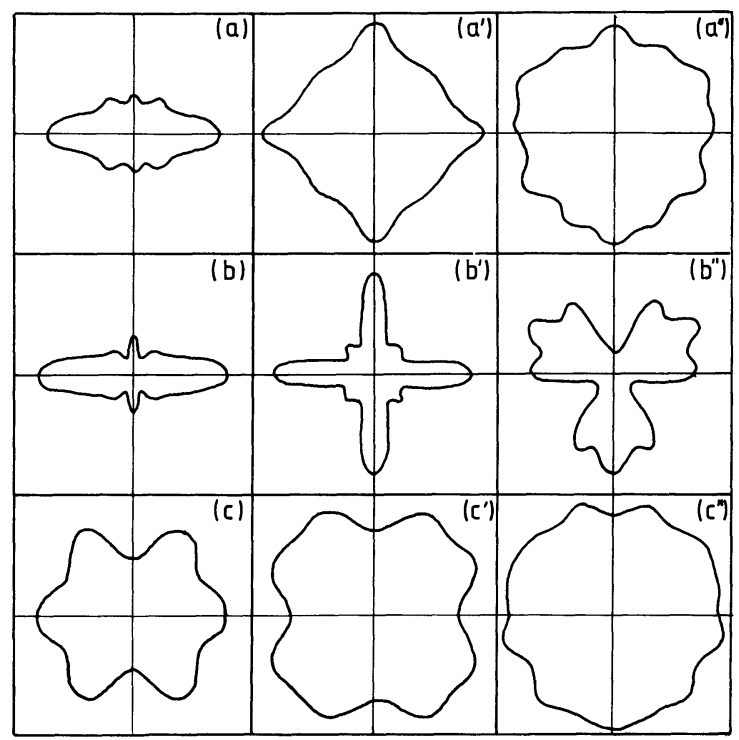

Fig. 3. - Polar plots of the theoretical azimuthal dependence of $W(110)(a, b, c), W(100)\left(a^{\prime}, b^{\prime}, c^{\prime}\right)$ and $W(111)$ $\left(a^{\prime \prime}, b^{\prime \prime}, c^{\prime \prime}\right) 4 f_{7 / 2}$ core photoelectron intensities for the total $\left(a, a^{\prime}, a^{\prime \prime}\right)$, bulk $\left(b, b^{\prime}, b^{\prime \prime}\right)$ and surface $\left(c, c^{\prime}, c^{\prime \prime}\right)$ emissions calculated from Kono et al. formula (s initial state) for the following conditions : $\theta=30^{\circ}, \alpha=22^{\circ} 5, h \nu=65 \mathrm{eV}$.

\section{Sensitivity to atomic surface displacements.}

It is well known that most of the transition metal surfaces undergo a surface relaxation, i.e. the first interlayer spacings differ from the values they would have in bulk metals. This effect is very weak for the most close-packed surfaces but may become significant for the open ones. On the other hand, in some experimental conditions (temperature, presence of adsorbates...) these surfaces may reconstruct. Thus, $\mathrm{W}(100)$ presents a $\mathrm{c}(2 \times 2)$ LEED pattern at low temperature which can be explained by alternate

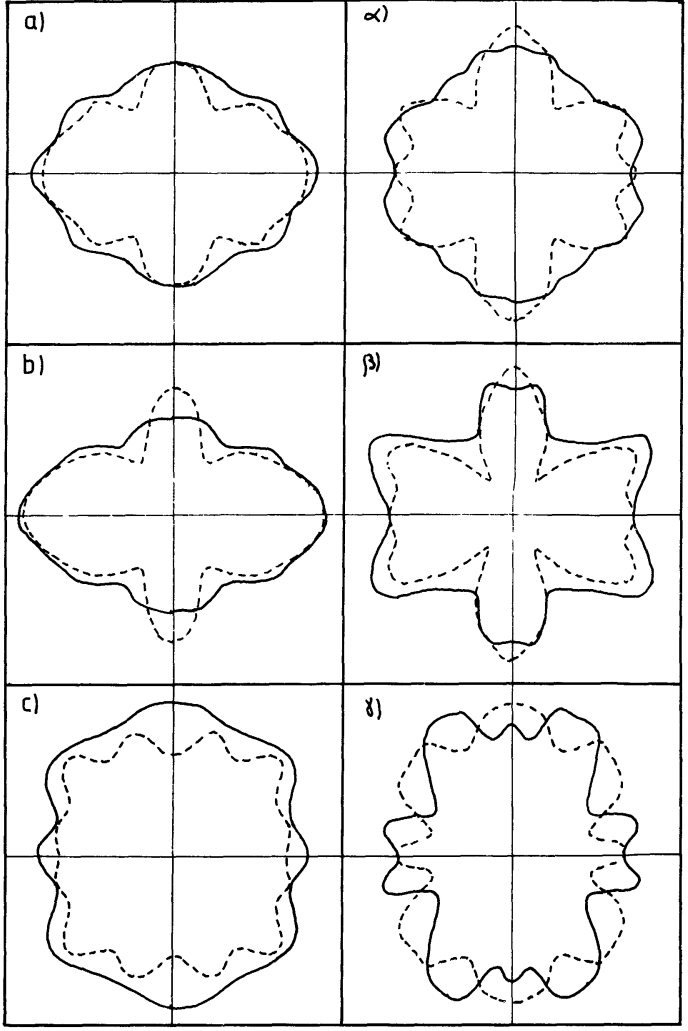

Fig. 4. - Polar plots of the theoretical azimuthal dependence of $\mathrm{W}(110) 4 \mathrm{f}_{7 / 2}$ core photoelectron intensities for the total $(\mathrm{a}, \alpha)$, bulk $(\mathrm{b}, \beta)$ and surface $(\mathrm{c}, \gamma)$ emissions calculated for an $\mathrm{f}$ initial state for two sets of parameters : - a, b, c : $\theta=30^{\circ}, \alpha=22^{\circ} 5, h \nu=65 \mathrm{eV}$ $-\alpha, \beta, \gamma: \theta=45^{\circ}, \alpha=20^{\circ}, h \nu=70 \mathrm{eV}$ [25] ---- unreconstructed surface ; $\longrightarrow$ reconstructed surface.

lateral displacements of surface atoms along the $\langle 11\rangle$ direction (« zig-zag » reconstruction [26, 27]). It has also been suggested that $\mathrm{W}(110)$ is not the perfect termination of the bulk but that the surface plane, while retaining its crystallographic structure, is rigidly displaced along the $\langle 10\rangle$ direction relative to the first underlayer [28]. Although this assertion is questionable for the clean surface, there are good reasons to admit that it is verified when hydrogen atoms are present at the surface [29]. Therefore, in the hope of using $\mathrm{PhD}$ as a tool to study clean surface crystallography, one should check its sensitivity to such surface atomic displacements.

Let us first present our results for the relaxation of the $(100)$ face of $W$. They are shown in figure $5 a, b$, $\mathrm{c}$, for three values of the relaxation: $0 \%, 5 \%$ (experimental value for $\mathrm{W}$ ), $10 \%$ (experimental value for $\mathrm{Ta}[30])$. Actually, the potentials and lattice parameters of $\mathrm{W}$ and $\mathrm{Ta}$ being very similar, our results can also be applied to the case of $\mathrm{Ta}$, for the same photoelectron kinetic energies. In view of this figure, the effect is too weak to be used as a tool for a precise determination of so small relaxations. 


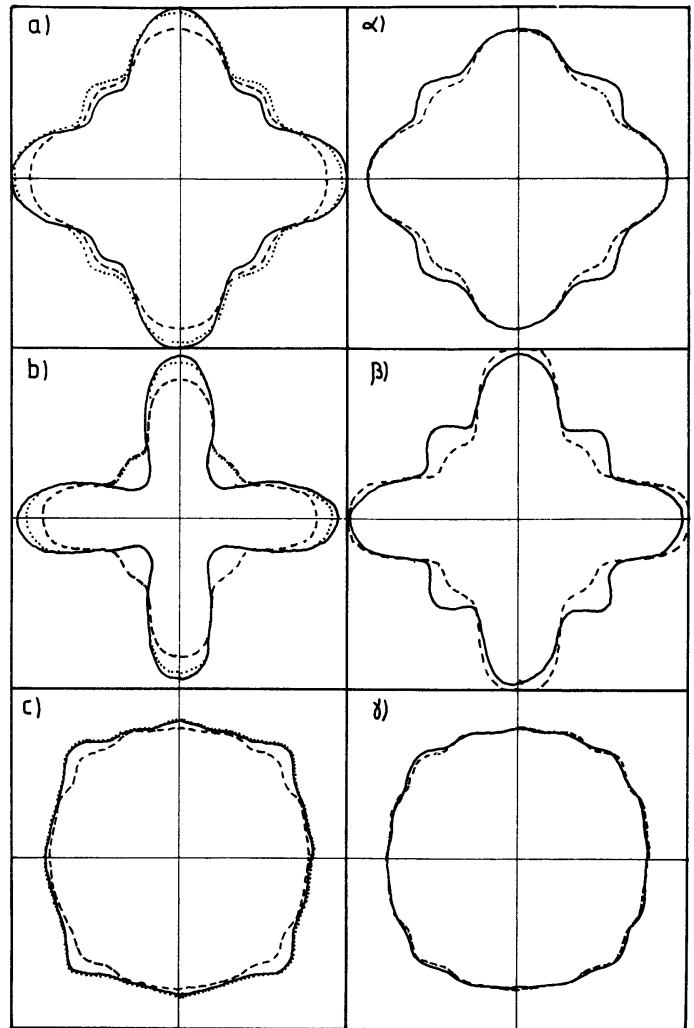

Fig. 5. - Influence of relaxation $(a, b, c)$ and zig-zag reconstruction $(\alpha, \beta, \gamma)$ on the theoretical azimuthal dependence of $\mathrm{W}(100) 4 \mathrm{f}_{712}$ core photoelectron intensities for the total $(\mathrm{a}, \alpha)$, bulk $(\mathrm{b}, \beta)$ and surface $(\mathrm{c}, \gamma)$ emissions calculated for an $\mathrm{f}$ initial state :

- a, b, c : - $10 \%$ relaxation ; ... $5 \%$ relaxation, --unrelaxed surface

$-\alpha, \beta, \gamma:-$ reconstructed surface ; --.- unreconstructed surface.

On the contrary, the bulk azimuthal pattern of $\mathrm{Ta}(100)$ is significantly modified by relaxation effects. This could be confirmed by comparing experimental results for Ta and $\mathrm{W}(100)$, for these conditions.

On the right hand side of the same figure, we have illustrated the influence of the "zig-zag" reconstruction on the azimuthal patterns of $\mathrm{W}(100)$ assuming a single domain of reconstruction. Herealso, the main difference can be seen on the bulk pattern in the four $\langle 11\rangle$ directions which all exhibit an enhanced lobe. Much less pronounced is the slight departure from fourfold symmetry which anyway cannot be observed experimentally due to the existence of several domains of reconstruction with perpendicular displacements. Contrary to the case of the relaxation, it is possible to measure the variation of the azimuthal patterns when the surface reconstructs by performing the experiment at room and nitrogen temperatures. The results will be presented and discussed in the next section.
Finally, we have considered also the influence of the possible reconstruction of the (110) face of $\mathrm{W}$. The results are shown in figure 4 for the two sets of experimental conditions mentioned in section 3 . The overall effect of the reconstruction is to smooth the structures of the azimuthal patterns. According to the most recent experiments, it appears that this reconstruction does not occur on a clean surface, but is triggered by a small amount of hydrogen [29]. The presence of these hydrogen atoms should not modify the calculated azimuthal patterns of the reconstructed face since hydrogen is a very weak scatterer. Therefore, it should be of greatest interest to perform the corresponding experiments.

\section{Experiments on $W(100)$.}

Angle resolved core level spectra are obtained using radiation from the $540 \mathrm{MeV}$ ACO storage ring at L.U.R.E. in Orsay. The chosen excitation energy $(h \nu=65 \mathrm{eV})$ is obtained from a high flux toroidal grating monochromator. Emission at $30^{\circ}$ from the normal of $\mathrm{W}(100)$ is collected to perform azimuthal photodiffraction scans. The energy resolution for the combined monochromator/analyser system is about $190 \mathrm{meV}$ whereas the angular resolution of the spherical analyser is better than $1^{\circ}$. The chamber and experimental equipment have been described elsewhere [11-13]. Here, two-axis rotations of the sample in situ are possible (polar angle $\theta$ and azimuthal angle $\varphi$, see Fig. 1a) with $\varphi$ rotations up to $220^{\circ}$. The angle $\alpha$ between the polarization vector $\hat{\varepsilon}$ and the surface normal is fixed at: $\alpha=22^{\circ} 5$ (p-like polarization). $\mathrm{W}(100) 4 \mathrm{f}_{7 / 2}$ level intensities are measured from a highly polished $\mathrm{W}$ surface, oriented to within $\pm 0.5^{\circ}$ of the (001) plane. The surface is prepared by argon ion sputtering, cycles of oxidization at $1500 \mathrm{~K}$, annealing at $2100 \mathrm{~K}$ and finally flashing up to $2400 \mathrm{~K}$. A high degree of surface order is verified by LEED : $p(1 \times 1)$ for the unreconstructed surface, $\mathrm{c}(2 \times 2)$ when the surface is reconstructed at low temperature $(\sim 90 \mathrm{~K})$. No impurity peaks were detected by Auger spectroscopy.

Let us recall that after subtracting a linear background each $4 f_{7 / 2}$ spectrum is decomposed into three peaks with Doniach-Sunjic lineshapes corresponding to the bulk (B), surface $\left(\mathrm{S}_{1}\right)$ and first underlayer $\left(\mathrm{S}_{2}\right)$ emissions. These peaks are then convoluted with the $190 \mathrm{meV}$-instrumental Gaussian function. The scattered intensities correspond to the areas of each component, normalized to the value of the secondary electron background taken at an energy sufficiently far from the $4 \mathrm{f}$ region to have a negligible azimuthal anisotropy. The corresponding results for the reconstructed and unreconstructed surfaces are shown in figure 6 . The raw data have been symmetrised with respect to $\langle 11\rangle$, then to $\langle 10\rangle$ axis, in 

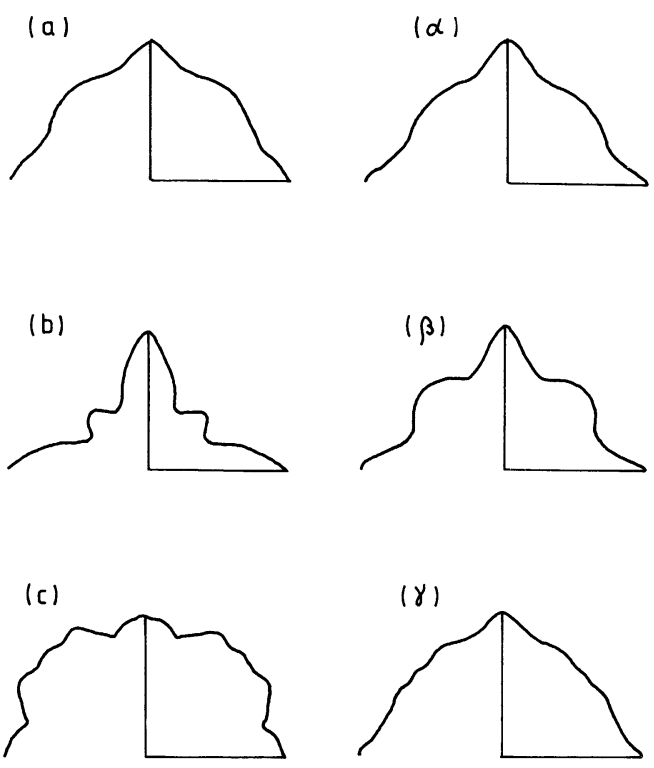

Fig. 6. - Polar plots of the experimental azimuthal dependence of $\mathrm{W}(100) 4 \mathrm{f}_{7 / 2}$ core photoelectron intensities for the total $(\mathrm{a}, \alpha)$, bulk $(\mathrm{b}, \beta)$ and surface $(\mathrm{c}, \gamma)$ emissions for the following conditions : $\theta=30^{\circ}, \alpha=22^{\circ} 5$, $h \nu=65 \mathrm{eV}$

- a, b, c : unreconstructed surface

$-\alpha, \beta, \gamma:$ thermally reconstructed surface.

accordance with the fourfold symmetry of both systems. For the unreconstructed surface, the agreement between experiments (Figs. 6a, b, c) and theory (Figs. 2a $a^{\prime} b^{\prime}, c^{\prime}$ and Figs. $3 a^{\prime}, b^{\prime}, c^{\prime}$ ) is excellent even when using an s-initial state. On the other hand, the influence of the reconstruction on the azimuthal patterns (Figs. $6 \alpha, \beta, \gamma$ ), which is particularly significant for bulk emission, distorts the four $\langle 11\rangle$ lobes in the same way as in the calculations (Figs. $5 \alpha, \beta, \gamma$ ). Finally, let us emphasize that the experiments have been performed twice with an excellent reproducibility.

\section{Discussion and conclusion.}

Our calculations have demonstrated that the use of an $\mathrm{s}$ initial state instead of the exact wave function in the case of a filled inner shell (with $\ell_{i}>0$ ), is quite hazardous even though good results can be obtained in some cases. However, the existence of two allowed transitions when $\ell_{i} \neq 0$ sets some problems since rigourously, the knowledge of the corresponding radial matrix elements is needed. This simplifies in the limits of low and high energies in which case the transitions towards $\ell_{i}-1$ and $\ell_{i}+1$ are respectively predominant. Unfortunately, the intermediate energy range in which both transitions are important is not very precisely delimited (see Appendix) and not very far from the energy used in the present study. Actually for $h \nu=65 \mathrm{eV}$ we have estimated $\tilde{\rho}_{k}(4,3) / \tilde{\rho}_{k}(2,3) \simeq 10^{-1} \quad$ whereas Goldberg et al. [31] predict 0.5 . It is thus interesting to investigate what would be obtained if we neglect $d$ wave and instead include $g$ wave final states. The results obtained using equation (20) with obvious changes are shown in figure 7 for the three low index faces of $\mathrm{W}$. The patterns are quite different from those of figure 2, although their intensities are quite alike, which justifies that the relative importance of the two transitions is indeed governed by $\tilde{\rho}_{k}\left(\ell, \ell_{i}\right)$. The comparison with experimental data in the case of W(100) (see Figs. 6a, b, c) clearly confirms that the transition $\mathrm{f} \rightarrow \mathrm{d}$ is predominant at this energy. Thus it would be of great interest to perform experiments with increasing photon energies from $h \nu=65 \mathrm{eV}$. The comparison with the calculated patterns should give a means to precise the energy dependence of $\tilde{\rho}_{k}(4,3) / \tilde{\rho}_{k}(2,3)$.

As a conclusion, we have shown (at least for $\mathrm{W}(100)$ ) that a single scattering treatment of photoelectron diffraction is sufficient to account for the observed azimuthal patterns, even in the kinetic energy range $\left(E_{\mathrm{c}} \sim 30 \mathrm{eV}\right)$ for which bulk and surface emissions are well separated. Moreover, in view of our results on the reconstruction of $\mathrm{W}(100)$, we can be optimistic in the use of photoelectron diffraction to study clean surface atom rearrangements.

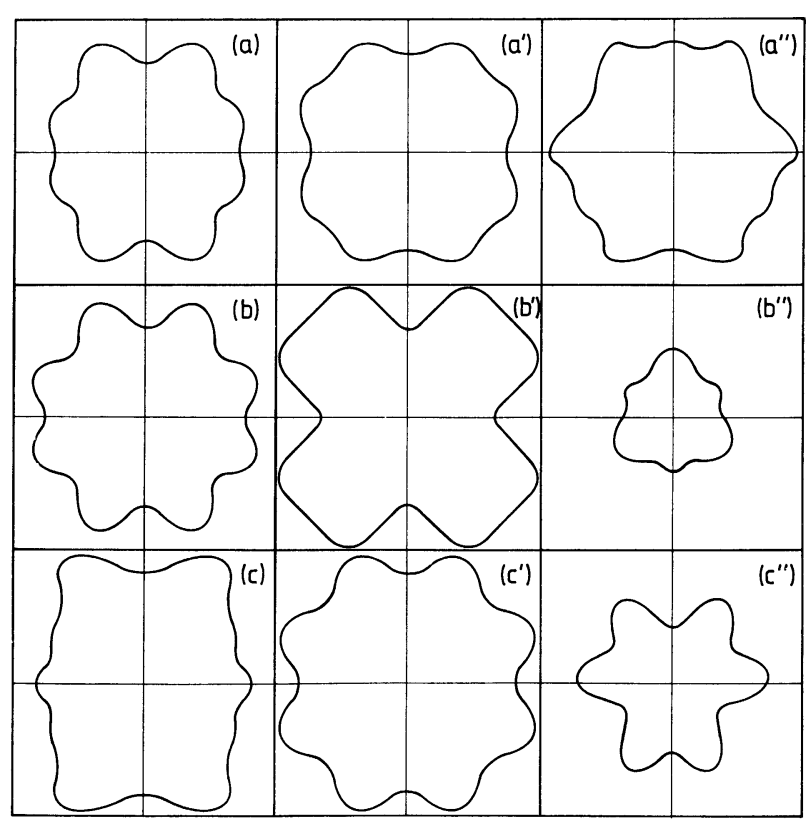

Fig. 7. - Polar plots of the theoretical azimuthal dependence of W(110): a, b, c, W(100): $\mathbf{a}^{\prime}, \mathrm{b}^{\prime}, \mathrm{c}^{\prime}$ and $\mathrm{W}(111)$ : $a^{\prime \prime}, b^{\prime \prime}, c^{\prime \prime} 4 f_{7 / 2}$ core photoelectron intensities for the total (a, $\left.a^{\prime}, a^{\prime \prime}\right)$, bulk (b, $\left.b^{\prime}, b^{\prime \prime}\right)$ and surface $\left(c, c^{\prime}, c^{\prime \prime}\right)$ emissions, calculated from Fujikawa formula (neglecting the $\mathrm{f} \rightarrow \mathrm{d}$ transition) for the following conditions: $\theta=30^{\circ}$, $\alpha=22^{\circ} 5, h \nu=65 \mathrm{eV}$. 
Finally, it would be highly desirable to perform experiments on other faces of $\mathrm{W}$ and other metals in order to precise to what extent our findings are general. On the other hand, it should be useful to check the validity of the plane wave approximation by performing a calculation with spherical waves (Eq. (13)). Some work is currently done in these directions.

\section{Acknowledgements.}

We are indebted to C. Noguera for enlightening discussions and to one of the referees for having drawn our attention on the work of Goldberg et al. (Ref. [31]) and for interesting suggestions.

\section{Appendix.}

CALCUlation OF RADIAL MATRIX ElEMENTS. The radial matrix elements are given by :

$\tilde{\rho}_{k}\left(\ell, \ell_{i}\right)=\int \mathcal{R}_{\ell}(k r) R_{\ell_{i}}(r) r^{3} \mathrm{~d} r$ with $\quad \ell=\ell_{i} \pm 1$.

For the core wave functions we have taken a Slater orbital [32] :

$$
R_{\ell_{i}}(r)=\frac{(2 \alpha)^{\frac{2 n^{*}+1}{2}}}{\sqrt{\Gamma\left(2 n^{*}+1\right)}} r^{n^{*}-1} \mathrm{e}^{-\alpha r}
$$

where $\Gamma$ is the gamma function.

For a W 4f level : $\ell_{i}=3, n^{*}=3.7, \alpha=6.34$. The final wave function is approximated by a plane wave so that :

$$
\begin{aligned}
\mathcal{R}_{\ell}(k r) & =\sqrt{\frac{k}{\pi}} j_{\ell}(k r) \\
& =\frac{1}{\sqrt{2 r}} J_{\ell}+\frac{1}{2}(k r)
\end{aligned}
$$

where the normalization of continuum wave functions per unit energy range is used [33]. Using [34] :

$$
\begin{aligned}
& \int_{0}^{\infty} \mathrm{e}^{-\alpha x} J_{\nu}(\beta x) x^{\mu-1} \mathrm{~d} x= \\
&=\frac{\left(\frac{\beta}{2 \alpha}\right)^{\nu} \Gamma(\nu+\mu)}{\alpha^{\mu} \Gamma(\nu+1)} \times \\
& \times F\left(\frac{\mu+\nu}{2}, \frac{\mu+\nu+1}{2}, \nu+1,-\frac{\beta^{2}}{\alpha^{2}}\right)
\end{aligned}
$$

where $F$ is the hypergeometric function, one finds :

$$
\begin{gathered}
\tilde{\rho}_{k}(\ell, 3)=\frac{2^{n^{*}-\ell-\frac{1}{2}}}{\alpha^{\ell+\frac{5}{2}}} k{ }^{\left(\ell+\frac{1}{2}\right)} \frac{\Gamma\left(\ell+3+n^{*}\right)}{\Gamma\left(\ell+\frac{3}{2}\right)} \times \\
\times \frac{F\left(\frac{\ell+3+n^{*}}{2}, \frac{\ell+4+n^{*}}{2}, \ell+\frac{3}{2},-\frac{k^{2}}{\alpha^{2}}\right)}{\sqrt{\Gamma\left(2 n^{*}+1\right)}}
\end{gathered}
$$

$\tilde{\rho}_{k}(2,3)$ and $\tilde{\rho}_{k}(4,3)$ are plotted in figure $\mathrm{A}(\mathrm{a})$ as a function of the photon energy. At the energy used in the present study $(h \nu=65 \mathrm{eV}) \quad \tilde{\rho}_{k}(4,3) /$ $\tilde{\rho}_{k}(2,3) \simeq 10^{-1}$. For comparison we have also plotted in figure $A(b)$, the same radial matrix elements calculated by Goldberg et al. [31] using a central field potential and the discrete orbitals $\psi_{n \ell_{m}}$ calculated by Hermann and Skillmann. The maxima of both curves are displaced towards lower photon energies. Nevertheless $\tilde{\rho}_{k}(2,3) / \tilde{\rho}_{k}(4,3)$ remains smaller than one for $h \nu=65 \mathrm{eV}(\simeq 0.5)$. Note that fitting a Slater type orbital to the corresponding Hermann-Skillmann wave function does not change our results significantly. Therefore, the difference with the calculations of Goldberg et al. comes from the final wave function which, in both cases, is rather approximated.

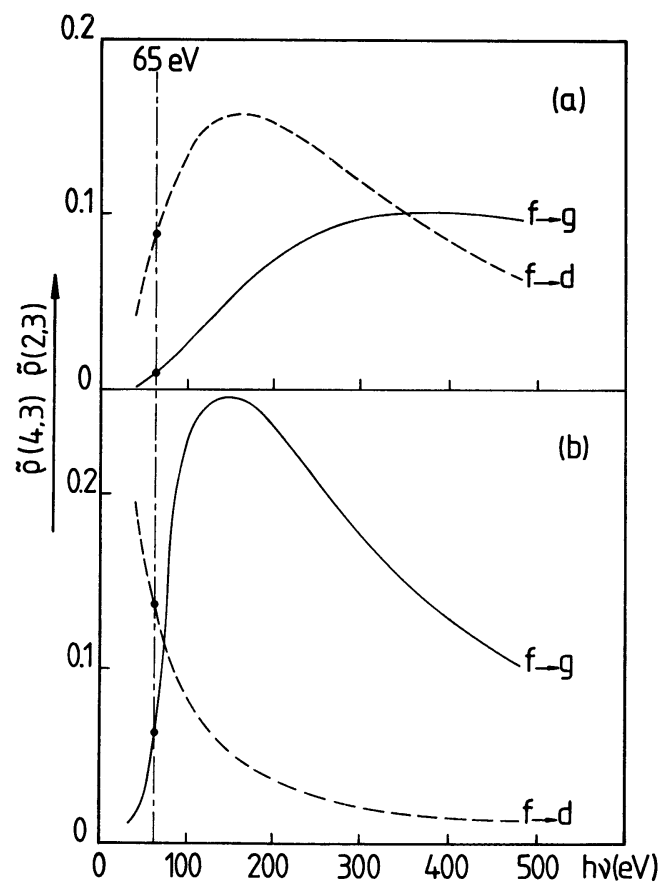

Fig. A. - Radial dipole matrix elements for the transition $\mathrm{f} \rightarrow \mathrm{d}\left(\tilde{\rho}_{k}(2,3)\right): \ldots$ and $\mathrm{f} \rightarrow \mathrm{g}\left(\tilde{\rho}_{\mathrm{k}}(4,3)\right):-$ in the case of $\mathrm{W}$ as a function of the photon energy : (a) using a plane wave approximation, (b) from Goldberg et al. [31]. 


\section{References}

[1] Woodruff, D. P., Norman, D., Holland, B. W., Smith, N. V., Farrell, H. H. and Traum, M. M., Phys. Rev. Lett. 41 (1978) 1130.

[2] Kevan, S. D., Rosenblatt, D. H., Benley, D., Lu, B. C. and Shirley, D. A., Phys. Rev. Lett. 41 (1978) 1565.

[3] Kono, S., Goldberg, S. M., Hall, N. F. T. and Fadley, C. S., Phys. Rev. Lett. 41 (1978) 117.

[4] Citrin, P. H., Wertheim, G. K. and Baer, Y., Phys. Rev. Lett. 41 (1978) 1425.

[5] Tran Minh Duc, Guillot, C., Lassailly, Y., LeCANTE, J., Jugnet, Y. and Védrine, J. C., Phys. Rev. Lett. 43 (1979) 789.

[6] Desjonquères, M. C., SpanjaARd, D., LASSAIlly, Y. and Guillot, C., Solid State Commun. 34 (1980) 807.

[7] VAN DER Veen, J. F., Himpsel, F. J. and EAstman, D. E., Phys. Rev. Lett. 44 (1980) 189.

[8] VAN DER Veen, J. F., Himpsel, F. J. and EAstman, D. E., Solid State Commun. 37 (1981) 555.

[9] VAN DER VeEn, J. F., Himpsel, F. J. and EAstMan, D. E., Solid State Commun. 40 (1980) 57.

[10] Tréglia, G., Desjonquères, M. C., SpanjaArd, D., Lassailly, Y., Guillot, C., Jugnet, Y., Tran Minh Duc and Lecante, J., J. Phys. $C$ 14 (1981) 3463.

[11] Guillot, C., Thuault, C., Jugnet, Y., Hoogewiss, R., Lecante, J., Tran Minh Duc, Tréglia, G., DesJonquères, M. C. and SpanjaArd, D., J. Phys. C 15 (1982) 4023.

[12] Guillot, C., Roubin, P., Lecante, J., DesjonQuères, M. C., Tréglia, G., SpanjaArd, D. and Jugnet, Y., Phys. Rev. B 30 (1984) 5487.

[13] Guillot, C., Chauveau, D., Roubin, P., Lecante, J., Desjonguères, M. C., Tréglia, G. and SPANJAARD, D., Surf. Sci. 162 (1985) 46.

[14] Liebsch, A., Phys. Rev. Lett. 32 (1974) 1203 ; Liebsch, A., Phys. Rev. B 13 (1976) 544.

[15] Kono, S., Goldberg, S. M., Hall, N. F. T. and FADley, C. S., Phys. Rev. B 22 (1980) 6085.

[16] MCDonnell, L., WoodrufF, D. P. and Holland, B. W., Surf. Sci. 51 (1975) 249.

[17] Lee, P. A., Phys. Rev. B 13 (1976) 5261.
[18] Fujikawa, T., J. Phys. Soc. Jpn 50 (1981) 1321 ; ibid. 51 (1982) 251 ; ibid. 54 (1985) 2747.

[19] Pendry, J. B., Low energy electron diffraction (Academic Press London and New York) 1974.

[20] Ziman, J. M., Elements of advanced quantum theory (Cambridge University Press) 1969.

[21] Sagurton, M., Bullock, E. L. and Fadley, C. S., Surf. Sci. 182 (1987) 287.

[22] Edmonds, A. R., Angular Momentum in Quantum Mechanics (Princeton University Press, Princeton) 1960.

[23] Christensen, N. E. and Feuerbacher, B., Phys. Rev. B 10 (1974) 2349.

[24] Sébilleau, D., Desjonquères, M. C., Chauveau, D., Guillot, C., Lecante, J., Tréglia, G. and SPANJAARD, D., Surf. Sci. 185 (1987) L 527.

[25] Tran Minh Duc, Spanjaard, D., Lecante, J., DesJonquères, M. C., Guillot, C., Jugnet, Y. and LASSAILly, Y., Proceedings of the fourth International conference on solid surfaces and the third European conference on surface science. Le Vide, Les couches minces, 201 (1980) 1204.

[26] KING, D. A., Phys. Scrip. 4 (1983) 34 and references therein.

[27] Legrand, B., Tréglia, G., Desjonquères, M. C. and Spanjaard, D., J. Phys. C. 19 (1986) 4463 and references therein.

[28] Van Hove, M. A. and Tong, S. Y., Surf. Sci. 54 (1976) 91.

[29] Chung, J. W., Ying, S. C. and Estrup, P. J., Phys. Rev. Lett. 56 (1986) 749.

[30] Titov, A. and Moritz, W., Surf. Sci. 123 (1982) L 709.

[31] Goldberg, S. M., Fadley, C. S. and Kono, S., J. Electron. Spectrosc. Relat. Phenom. 21 (1981) 285.

[32] Slater, J. C., Phys. Rev. 36 (1930) 57.

[33] Bethe, H. A. and SAlPeter, E. E., Quantum Mechanics of one and two electron atoms (Springer Verlag, Berlin) 1957, 23.

[34] Gradshteyn, I. S. and RyzhIK, I. M., Tables of integrals, series and products (Academic Press, New York) 1980, 711. 